\title{
Affinity and Distance. On the Newtonian Structure of Some Data Kernels
}

https://doi.org/10.1515/agms-2018-0005

Received August 15, 2017; revised April 6, 2018; accepted April 23, 2018

\begin{abstract}
Let $X$ be a set. Let $K(x, y)>0$ be a measure of the affinity between the data points $x$ and $y$. We prove that $K$ has the structure of a Newtonian potential $K(x, y)=\varphi(d(x, y))$ with $\varphi$ decreasing and $d$ a quasi-metric on $X$ under two mild conditions on $K$. The first is that the affinity of each $x$ to itself is infinite and that for $x \neq y$ the affinity is positive and finite. The second is a quantitative transitivity; if the affinity between $x$ and $y$ is larger than $\lambda>0$ and the affinity of $y$ and $z$ is also larger than $\lambda$, then the affinity between $x$ and $z$ is larger than $v(\lambda)$. The function $v$ is concave, increasing, continuous from $\mathbb{R}^{+}$onto $\mathbb{R}^{+}$with $v(\lambda)<\lambda$ for every $\lambda>0$.
\end{abstract}

Keywords: uniform spaces; metric spaces; affinity kernel

MSC: Primary 54E15, 54E35, 31E99

\section{Introduction}

The notion of affinity, when applied to a data set $X$ involves an empirical construction of a real valued kernel $K$ defined on $X \times X$ reflecting some similarity of any two data points $x$ and $y$ in $X$, given by particular features shared by $x$ and $y$. Of course, since the features to be considered relevant depend on the particular situation and even on the points of view of the observer, the notion of affinity becomes healthily wide. Even so, some basic properties seen to be shared by such diversity of affinity kernels. These properties are the symmetry, $K(x, y)=K(y, x)$, and the positive definiteness, i.e., for every finite subset $F$ of $X$ the matrix $(K(x, y): x \in$ $F, y \in F)$ is positive semi-definite. Precisely, the inequality $\sum_{x, y \in F} \xi_{x} \xi_{y} K(x, y) \geq 0$ holds for every $F \subset X$ with $\#(F)<\infty$ and every choice of $\xi_{x}, \xi_{y} \in \mathbb{R}$. Of course the most elementary, but useless, affinity is a diagonal kernel, $K(x, x)>0, K(x, y)=0$ for $x \neq y$. On the other hand, several realistic models contain a natural weak transitivity which can be seen as a deconcentration of the affinity outside the diagonal of $X \times X$. As a toy example, assume that $x$ and $y$ are two individuals, in a homogeneous cultural context, and $K(x, y)$ counts the number of common watched movies by $x$ and $y$ during the last decade. Usually $K(x, y)$ is much smaller than the total number of available movies in that period, then $K(x, y)$ reflects that the individuals $x$ and $y$ share some criteria for their film genres choice. On the other hand, if $z$ is a third individual in the same social group that shares with $y$ some criteria for their film genres choice, then $x$ and $z$ must share some weakened preferences. For example, if $K(x, y)>100$ and $K(y, z)>100$ then $K(x, z)>50$. What is remarkable is that these type of transitivity is satisfied by some very classical kernels as is the Newtonian potential. Before going to this central point in our approach, let us briefly review some particular kernel which have been extensively used. For example in the detection of similarity of documents, the cosine affinity kernel $K(x, y)=\frac{\langle x, y\rangle}{\|x\|\|y\|}$ becomes natural. Here $x$ and $y$ are vectors in a data set $X$ contained in a Hilbert space $H$ (for example $\mathbb{R}^{N}, N$

\footnotetext{
Hugo Aimar: Instituto de Matemática Aplicada del Litoral, UNL, CONICET, FIQ. CCT-CONICET-Santa Fe, Predio "Dr. Alberto Cassano”, Colectora Ruta Nac. 168 km 0, Paraje El Pozo, S3007ABA Santa Fe, Argentina, E-mail: haimar@santafe-conicet.gov.ar ^Corresponding Author: Ivana Gómez: Instituto de Matemática Aplicada del Litoral, UNL, CONICET, FIQ. CCT-CONICET-Santa Fe, Predio “Dr. Alberto Cassano”, Colectora Ruta Nac. 168 km 0, Paraje El Pozo, S3007ABA Santa Fe, Argentina,

E-mail: ivanagomez@santafe-conicet.gov.ar
} 
large) and $\langle x, y\rangle$ is the scalar product in $H$. At a first glance the kernel $K(x, y)$ does not depend of a distance between $x$ and $y$. Nevertheless, after the projection of the data set $X$ on the unit sphere $\{\|x\|=1\}$ of $H$, we have that with $\xi=\frac{x}{\|x\|}$ and $\eta=\frac{y}{\|y\|}, K(\xi, \eta)=\langle\xi, \eta\rangle=\varphi(\|\xi-\eta\|)$ with $\varphi(t)=1-\frac{t^{2}}{2}$. In other words, affinity can be written in terms of a decreasing function of the Euclidean distance, when restricted to the unit sphere of $H$. Other usual types of affinity kernels are explicitly built in terms of known metrics, such is the case of the so called RBF-kernel (radial basis function) given by the bell $K_{R}(x, y)=e^{-R\|x-y\|^{2}}$. Here $R$ becomes large if we expect that the affinity between $x$ and $y$ becomes more demanding. On the other hand since the preservation of the Markov property along the family $K_{R}$ requires normalization, for $R$ large we have a Gauss-Weiertrass kernel with variance $\sigma^{2}=\frac{1}{R}$. See [4].

Let us observe that if $N(x, y)=\frac{1}{|x-y|}$ is the Newtonian potential for $x$ and $y$ in $\mathbb{R}^{3}$, then the triangle inequality $|x-z| \leq|x-y|+|y-z|$ shows that if for $\lambda>0$ we have $N(x, y)>\lambda$ and $N(y, z)>\lambda$, then we also have $N(x, z)>\frac{\lambda}{2}$, that, with $\lambda=100$, agrees with our heuristic transitivity for the example of movies preferences. In some sense, attending to the historical development of the ideas in classical mechanics, the data obtained by Tycho Brahe and organized by Johannes Kepler was finally understood (learnt?) by Isaac Newton who, after projection on space by elimination of the time variable (as in the case of the cosine affinity) gave such a simple attraction or affinity kernel $K(x, y)=N(x, y)=\frac{1}{|x-y|}$ as the basic descriptor the gravitational field. In this paper we start by the heuristic consideration of affinity as attraction. And we search for necessary and sufficient conditions on a positive and symmetric kernel $K(x, y)$ defined on a set $X$ in such a way that $K(x, y)=\varphi(d(x, y))$ (as $N(x, y)$ is the composition of $\varphi(s)=\frac{1}{s}$ and $\left.d(x, y)=|x-y|\right)$ with $\varphi$ decreasing, $\varphi(0)=+\infty, \varphi(+\infty)=0$ and $d$ a quasi-distance on $X$.

In [1] the authors give necessary and sufficient conditions on families of subsets of a set $X$ in such a way that these subsets are close to be balls of a quasi-metric on $X$. A similar situation, in terms of families of subsets of $X \times X$ is considered here, regarding the basic properties of quasi-metric stripes around the diagonal of $X \times X$. What is more important is that this characterization of quasi-metric bands can be used to provide a structure result of some affinity kernels.

Let $X$ be a set. Let $K(x, y)$ be a nonnegative number measuring the affinity between the two data points $x$ and $y$. We shall consider some basic properties of affinity which will be sufficient to obtain the Newtonian potential type structure for $K$. Symmetry; affinity is a symmetric relation on $X \times X(K(x, y)=K(y, x)$ for every $x, y \in X)$. Positivity; there is positive affinity between any couple of data points $x$ and $y(K(x, y) \geq 0$ for every $x, y \in X)$. Diagonal singularity; the self affinity is unimprovable. Precisely, the affinity of each data point $x$ with itself is $+\infty(K(x, x)=+\infty$ for every $x)$ but for $x \neq y$ the affinity is finite $(K(x, y)<\infty$ for $x \neq y)$. Quantitative Transitivity; if the affinity between the data points $x$ and $y$ is larger than $\lambda>0$ and the affinity between $y$ and $z$ is larger than $\lambda$ then the affinity between the points $x$ and $z$ is larger than $v(\lambda)$. Here $v$ is a nonnegative, concave, increasing and continuous function defined on $\mathbb{R}^{+}$onto $\mathbb{R}^{+}$such that $v(\lambda)<\lambda$.

A quasi-metric in $X$ is a nonnegative symmetric function $d$ defined on $X \times X$ which vanishes only on the diagonal $\Delta$ of $X \times X$ and satisfies a weak form of the triangle inequality, there exists a constant $\tau(\geq 1)$ such that the inequality $d(x, z) \leq \tau(d(x, y)+d(y, z))$ holds for every $x, y$ and $z$ in $X$.

The main result of this paper can be stated as follows.

Theorem 1.1. Let $X$ be a set. Let $K: X \times X \rightarrow \mathbb{R}^{+}$be a symmetric function satisfying the singularity and the quantitative transitivity conditions. Then there exist a decreasing and continuous function $\varphi$ defined in $\mathbb{R}^{+}$and a quasi-metric $d$ on $X$ such that

$$
K(x, y)=\varphi(d(x, y)) .
$$

Moreover, $d(x, y)=h(x, y) \rho(x, y)$ with $\rho$ a metric on $X$ and $h$ a symmetric function such that for some constants $0<c_{1}<c_{2}<\infty$ satisfies $c_{1} \leq h(x, y) \leq c_{2}$ for every $x$ and $y$ in $X$.

At this point, as one of our referees let us observe, it is important to notice that our hypothesis on $K$ can be rewritten in terms of $\frac{1}{K(x, y)}=:[x, y]$ by saying, as in Chittenden [3] $\S 12$, page 26, that $[x, y]$ is a uniformly regular ècart. That is, $[x, y]=0$ if and only if $x=y$ and there is a function $f(\lambda)=\frac{1}{v(\lambda)}$ such that $[x, y] \leq \lambda$ and $[z, y] \leq \lambda$ implies $[x, z] \leq f(\lambda)$. In [3] it is shown that the above conditions on $[x, z]$ are sufficient for 
metrizability of the underlying space. In some sense our result provides a quantitative structural version of this metrization theorem when $\frac{1}{f}$ is nonnegative, concave, increasing and continuous function defined on $\mathbb{R}^{+}$ with $f(\lambda)>\frac{1}{\lambda}$. Moreover we prove a Newtonian potential type structure for $\frac{1}{[x, y]}$.

The deepest results on the structure of quasi-metrics are due to Macias and Segovia and are contained in [8]. See also [2]. The most significant for our purposes is the fact that each quasi-metric is equivalent to a power of a metric. In other words, given a quasi-metric $d$ on $X$ with constant $\tau$, there exist $\beta \geq 1$ and a metric $\rho$ on $X$ such that for some positive constants $a_{1}$ and $a_{2}$ the inequalities

$$
a_{1} d(x, y) \leq \rho^{\beta}(x, y) \leq a_{2} d(x, y)
$$

hold for every $x$ and $y$ in $X$. Actually the proof is based in Frink's lemma of metrization of uniform structures with a countable basis ([6], [7]).

The rest of the paper is organized in the following way. Section 2 gives a characterization of quasi-metrics on a set in terms of properties of the family of stripes in $X \times X$ induced by the quasi-metric. Section 3 contains the construction of the monotonic profile. In Section 4 we prove the main result.

\section{Quasi-metrics and families of stripes around the diagonal}

Let $X$ be a set. The composition of two subsets $U$ and $V$ of $X \times X$ is given by $V \circ U=\{(x, z) \in X \times X$ : there exists $y \in X$ such that $(x, y) \in U$ and $(y, z) \in V\}$. A subset $U$ in $X \times X$ is said to be symmetric if $(x, y) \in U$ if and only if $(y, x) \in U$. Set $\Delta$ to denote the diagonal in $X \times X$, i.e. $\Delta=\{(x, x): x \in X\}$. When a quasi-metric $\delta$ with constant $\tau$ is given in $X$, it is easy to check that the one parameter family $\mathcal{V}(r)=\{(x, y) \in X \times X: \delta(x, y)<$ $r\} ; r>0$, of stripes around the diagonal of $X \times X$, satisfies

(S1) each $\mathcal{V}(r)$ is symmetric;

(S2) $\Delta \subseteq \mathcal{V}(r)$, for every $r>0$;

(S3) $\mathcal{V}\left(r_{1}\right) \subseteq \mathcal{V}\left(r_{2}\right)$, for $0<r_{1} \leq r_{2}$;

(S4) $\cup_{r>0} \mathcal{V}(r)=X \times X$;

(S5) $\cap_{r>0} \mathcal{V}(r) \subseteq \Delta$;

(S6) there exists $T \geq 1$ such that $\mathcal{V}(r) \circ \mathcal{V}(r) \subseteq \mathcal{V}(\operatorname{Tr})$, for every $r>0$.

Actually, the constant $T$ in (S6) can be taken to be the triangle constant $\tau$ of $\delta$. Set $\mathcal{P}(X \times X)$ to denote the set of subsets of $X \times X$.

Theorem 2.1. Let $\mathcal{V}: \mathbb{R}^{+} \rightarrow \mathcal{P}(X \times X)$ be a one parameter family of the subsets of $X \times X$ that satisfies (S1) to (S6) above. Then the function $\delta$ defined on $X \times X$ by $\delta(x, y)=\inf \{r>0:(x, y) \in \mathcal{V}(r)\}$ is a quasi-metric on $X$ with $\tau \leq$ T. Moreover, for each $\gamma>0$, we have

$$
\mathcal{V}_{\delta}(r) \subseteq \mathcal{V}(r) \subseteq \mathcal{V}_{\delta}((1+\gamma) r)
$$

hold for every $r>0$, where $\mathcal{V}_{\delta}(s)=\{(x, y) \in X \times X: \delta(x, y)<s\}$.

Proof. From (S4) for the family $v$ we see that $\delta(x, y)$ is well defined as a nonnegative real number. The symmetry of $\delta$ follows from (S1). The fact that $\delta$ vanishes on the diagonal $\Delta$ follows from $\Delta \subseteq \cap_{r>0} \mathcal{V}(r)$ which is contained in (S2). Now, if $(x, y) \in X \times X$ and $\delta(x, y)=0$, then, from (S3) for each $r>0,(x, y) \in \mathcal{V}(r)$. Now, from (S5) we necessarily have that $(x, y) \in \Delta$ or, in other words $x=y$. Let us check that $\delta$ satisfies a triangle inequality. Let $x, y$ and $z$ be three points in $X$. Let $\varepsilon>0$. Take $r_{1}>0$ and $r_{2}>0$ such that $r_{1}<\delta(x, y)+\varepsilon, r_{2}<\delta(y, z)+\varepsilon,(x, y) \in \mathcal{V}\left(r_{1}\right)$ and $(y, z) \in \mathcal{V}\left(r_{2}\right)$. From (S6) with $r^{\star}=\sup \left\{r_{1}, r_{2}\right\}$, we have $(x, z) \in \mathcal{V}\left(r_{2}\right) \circ \mathcal{V}\left(r_{1}\right) \subseteq \mathcal{V}\left(r^{*}\right) \circ \mathcal{V}\left(r^{*}\right) \subseteq \mathcal{V}\left(\operatorname{Tr}^{\star}\right)$. Hence $\delta(x, z) \leq T r^{\star} \leq T\left(r_{1}+r_{2}\right) \leq T(\delta(x, y)+\delta(y, z))+2 \varepsilon T$ and we get the triangle inequality with $\tau=T$. Notice first that from (S3), $\nu_{\delta}(r) \subseteq \mathcal{V}(r)$ for every $r>0$. Take now $(x, y) \in \mathcal{V}(r)$, then $\delta(x, y) \leq r<(1+\gamma) r$, so that $\mathcal{V}(r) \subseteq \mathcal{V}_{\delta}((1+\gamma) r)$ for every $\gamma>0$ and every $r>0$. 
The next result follows from the above and the metrization theorem of quasi-metric spaces proved in [8] as an application of Frink's Lemma on metrizability of uniform spaces with countable bases.

Theorem 2.2. Let $\mathcal{V}$ and $\delta$ be as in Theorem 2.1. Then, there exist a constant $\beta \geq 1$ and a metric $\rho$ on $X$ such that

(i) $4^{-\beta} \delta \leq \rho^{\beta} \leq 2^{\beta} \delta$;

(ii) $\mathcal{V}_{\rho^{\beta}}\left(\frac{r}{4^{\beta}}\right) \subseteq \mathcal{V}(r) \subseteq \mathcal{V}_{\rho^{\beta}}\left(2^{\beta+1} r\right)$ where $\mathcal{V}_{\rho^{\beta}}(r)=\left\{(x, y) \in X \times X: \rho^{\beta}(x, y)<r\right\}$.

Proof. Following the proof of Theorem 2 on page 261 in [8] take $\alpha<1$ such that $\left(3 T^{2}\right)^{\alpha}=2$ and $\beta=\frac{1}{\alpha}>1$. With $\rho$ the metric provided by the metrization theorem for uniform spaces with countable bases, we have that $4 \rho(x, y) \geq \delta(x, y)^{\alpha} \geq \frac{1}{2} \rho(x, y)$. So that

$$
\frac{1}{4^{\beta}} \delta(x, y) \leq \rho(x, y)^{\beta} \leq 2^{\beta} \delta(x, y),
$$

and (ii) follow from these inequalities and (2.1) with $\gamma=1$.

\section{Building the basic profile shape}

The classical inverse proportionality to the square of the distance between the two bodies for the gravitation field, translates into inverse proportionality to the distance for the potential. That is $\varphi(r)=\frac{1}{r}$ for the gravitational potential.

This section is devoted to the construction of the basic shapes of the profiles that allow the Newtonian representation of the kernels. This construction requires to solve a functional inequality involving the function $v$ that controls the quantitative transitive property of $K$.

Proposition 3.1. Let $v$ be a concave, continuous, nonnegative and increasing function defined on $\mathbb{R}^{+}$onto $\mathbb{R}^{+}$ such that $v(\lambda)<\lambda$ for every $\lambda>0$. Then, given $M>1$, there exists a continuous, decreasing and convex function $\psi$ defined on $\mathbb{R}^{+}$with $\psi(1)=1$ such that the inequality

$$
\psi(\nu(\lambda)) \leq M \psi(\lambda)
$$

holds for every $\lambda>0$.

Proof. Set $\lambda_{0}=1, \lambda_{1}=v(1)$ and $\lambda_{-1}=v^{-1}$ (1). Notice that $\lambda_{1}<1$ and $\lambda_{-1}>1$. In fact, $\lambda_{1}=v(1)<1$ and $1=v^{-1}(v(1))=v^{-1}\left(\lambda_{1}\right)<v^{-1}(1)=\lambda_{-1}$. Set for $k \in \mathbb{N}, \lambda_{k}=v\left(\lambda_{k-1}\right)$ and $\lambda_{-k}=v^{-1}\left(\lambda_{-k+1}\right)$. Notice that $\lambda_{k}$ decreases as $k \rightarrow \infty$ and increases when $k \rightarrow-\infty$. The continuity of $v$ and the property $v(\lambda)<\lambda$ for every positive $\lambda$ imply that $\lambda_{k} \rightarrow 0$ as $k \rightarrow \infty$ and $\lambda_{k} \rightarrow \infty$ as $k \rightarrow-\infty$ monotonically. This basic sequence $\left\{\lambda_{k}: k \in \mathbb{Z}\right\}$ allows to construct a function $\psi$ in the following way. Set $\psi\left(\lambda_{k}\right)=M^{k}, k \in \mathbb{Z}$ and for $\lambda \in\left[\lambda_{k+1}, \lambda_{k}\right]$ define $\psi(\lambda)$ by linear interpolation. It is clear that $\psi$ is continuous, decreasing, $\psi\left(0^{+}\right)=+\infty, \psi(\infty)=0$, $\psi(1)=\psi\left(\lambda_{0}\right)=M^{0}=1$ and that $\psi$ is convex. We only have to check that $\psi$ solves inequality (3.1). On the sequence $\left\{\lambda_{k}: k \in \mathbb{Z}\right\}$, (3.1) becomes an equality. In fact, $\psi\left(v\left(\lambda_{k}\right)\right)=\psi\left(\lambda_{k+1}\right)=M^{k+1}=M M^{k}=M \psi\left(\lambda_{k}\right)$.

Let us now take $\lambda \in\left(\lambda_{k+1}, \lambda_{k}\right)$ for $k \in \mathbb{Z}$. For such $\lambda, \psi(\lambda)$ satisfies

$$
\frac{M^{k+1}-M^{k}}{\lambda_{k}-\lambda_{k+1}}=\frac{M^{k+1}-\psi(\lambda)}{\lambda-\lambda_{k+1}} \text {. }
$$

On the other hand, since $\lambda_{k+1}<\lambda<\lambda_{k}$, we have that $\lambda_{k+2}=v\left(\lambda_{k+1}\right)<v(\lambda)<v\left(\lambda_{k}\right)=\lambda_{k+1}$. Hence $\psi(v(\lambda))$ satisfies

From (3.2) and (3.3) we get

$$
\frac{M^{k+2}-M^{k+1}}{\lambda_{k+1}-\lambda_{k+2}}=\frac{M^{k+2}-\psi(v(\lambda))}{v(\lambda)-\lambda_{k+2}} .
$$

$$
\psi(\lambda)=M^{k+1}-M^{k}(M-1) \frac{\lambda-\lambda_{k+1}}{\lambda_{k}-\lambda_{k+1}}
$$


and

$$
\psi(v(\lambda))=M\left(M^{k+1}-M^{k}(M-1) \frac{v(\lambda)-\lambda_{k+2}}{\lambda_{k+1}-\lambda_{k+2}}\right) .
$$

Now, since $v$ is concave, we have for $\lambda_{k+1}<\lambda<\lambda_{k}$ that

$$
\frac{v(\lambda)-v\left(\lambda_{k+1}\right)}{\lambda-\lambda_{k+1}} \geq \frac{v\left(\lambda_{k}\right)-v\left(\lambda_{k+1}\right)}{\lambda_{k}-\lambda_{k+1}},
$$

hence, $\psi(v(\lambda)) \leq M \psi(\lambda)$.

The basic shapes for the profiles $\varphi$ in our main result will be given as composition of the inverse $\eta$ of $\psi$ with power laws.

\section{Proof of the main result}

Let us start by rewriting, formally, the properties of symmetry, positivity, singularity and transitivity of a data affinity kernel $K(x, y)$ defined on the set $X \times X$. Let $K: X \times X \rightarrow \mathbb{R}$ such that

(K1) $K(x, y)=K(y, x)$, for every $x$ and $y$ in $X$;

(K2) $K(x, y)>0$, for every $x$ and $y$ in $X$;

(K3) $K(x, y)=+\infty$ if and only if $x=y$;

(K4) there exists a continuous, concave, increasing and nonnegative function $v$ defined on $\mathbb{R}^{+}$onto $\mathbb{R}^{+}$, with $v(\lambda)\langle\lambda, \lambda>0$, such that $K(x, z)>v(\lambda)$ whenever there exists $y \in X$ with $K(x, y)>\lambda$ and $K(y, z)>\lambda$, for every $\lambda>0$.

With these properties, Theorem 1.1 can be restated as follows.

Theorem 4.1. Let $X$ be a set. Let $K$ be a kernel on $X \times X$ satisfying properties(K1) to (K4). Then, there exist a metric $\rho$ on $X$, a real number $\beta \geq 1$, a function $h(x, y)$ defined on $X \times X$ with $2^{-1 / \beta} \leq h(x, y) \leq 4$ and a function $\varphi: \mathbb{R}^{+} \rightarrow \mathbb{R}^{+}$continuous, decreasing with $\varphi\left(0^{+}\right)=+\infty$ and $\varphi(\infty)=0$, such that

$$
K(x, y)=\varphi(h(x, y) \rho(x, y)) .
$$

Proof. Let $v$ be the function provided by (K4). Let $\psi$ be given by Proposition 3.1 with this function $v$, and $M=2$. Hence $\psi(v(\lambda)) \leq 2 \psi(\lambda)$ for every $\lambda>0$. Take $\eta=\psi^{-1}$ and $\nu: \mathbb{R}^{+} \rightarrow \mathcal{P}(X \times X)$ given by

$$
\mathcal{V}(r)=E_{\eta(r)}=\{(x, y): K(x, y)>\eta(r)\} .
$$

Let is check that $\mathcal{V}$ satisfies properties (S1) to (S6) in Section 2 with constant $T=2(=M)$. From (K1) we see that each $E_{\lambda}$ is symmetric, in particular $\mathcal{V}(r)$ is symmetric for every $r>0$. Since $K(x, x)=+\infty$, from (K3), we have that $\Delta \subseteq E_{\eta(r)}=\mathcal{V}(r)$ for $r>0$. In order to check (S3) take $0<r_{1}<r_{2}<\infty$. Hence $\eta\left(r_{1}\right)>\eta\left(r_{2}\right)$, so that $K(x, y)>\eta\left(r_{1}\right)$ implies $K(x, y)>\eta\left(r_{2}\right)$. Or, in other words $E_{\eta\left(r_{1}\right)} \subset E_{\eta\left(r_{2}\right)}$. Or $\mathcal{V}\left(r_{1}\right) \subseteq \mathcal{V}\left(r_{2}\right)$. The positivity (K2) of $K(x, y)$ shows (S4). Property (S5) of $\mathcal{V}$ follows from (S3). To prove (S6) for $\mathcal{V}$, take $r>0$. If $(x, z) \in \mathcal{V}(r) \circ \mathcal{V}(r)=$ $E_{\eta(r)} \circ E_{\eta(r)}$, then there exists $y \in X$ such that $K(x, y)>\eta(r)$ and $K(y, z)>\eta(r)$. From (K4), $K(x, z)>v(\eta(r))$. Now applying (3.1) with $\lambda=\psi^{-1}(r)$ we get $K(x, z)>v\left(\psi^{-1}(r)\right) \geq \eta(2 r)$, or $(x, z) \in E_{\eta(2 r)}=\mathcal{V}(2 r)$. Hence (S6) for $\mathcal{V}$ holds with $T=2$. We can, then apply the results of Section 2. First to produce a quasi-metric $\delta$ as in Theorem 2.1 and then to provide the metric $\rho$ and the exponent $\beta$ given in Theorem 2.2. Thus, for every $r>0$, $\mathcal{V}_{\rho^{\beta}}\left(\frac{r}{4^{\beta}}\right) \subseteq \mathcal{V}(r)=E_{\eta(r)} \subseteq \mathcal{V}_{\rho^{\beta}}\left(2^{\beta+1} r\right)$, where $\rho$ is a metric in $X$ and, since $T$ can be taken to be equal to 2, $\beta=\log _{2} 12$ works. The above inclusions, taking $s=r^{1 / \beta}$, are equivalent to

$$
\left\{\rho<\frac{s}{4}\right\} \subseteq\left\{(\psi \circ K)^{1 / \beta}>s\right\} \subseteq\left\{\rho<2^{1+1 / \beta} s\right\}
$$

for every $s>0$. Let $x$ and $y$ be two different points in $X$. Since $0<K(x, y)<\infty$ so is $(\psi \circ K)^{1 / \beta}(x, y)$. There exists, then, a unique $j \in \mathbb{Z}(j=j(x, y))$ such that $2^{j} \leq(\psi \circ K)^{1 / \beta}(x, y)<2^{j+1}$. By the second inclusion in (4.1) 
we see that $\rho(x, y)<2^{1 / \beta} 2^{j} \leq 2^{1 / \beta}(\psi \circ K)^{1 / \beta}(x, y)$. On the other hand, since $(\psi \circ K)^{1 / \beta}(x, y)<2^{j+1}$, from the first inclusion in (4.1) we necessarily have that $\rho(x, y) \geq \frac{2^{j+1}}{4}>\frac{1}{4}(\psi \circ K)^{1 / \beta}(x, y)$. Hence for $x \neq y$ we have

$$
\frac{1}{4}(\psi \circ K)^{1 / \beta} \leq \rho \leq 2^{1 / \beta}(\psi \circ K)^{1 / \beta} .
$$

Set $h(x, y)=\frac{(\psi \circ K)^{1 / \beta}}{\rho(x, y)}$ for $x \neq y$ and $h(x, x)=1$. Then $\frac{1}{2^{1 / \beta}} \leq h \leq 4$ and $K(x, y)=\psi^{-1}\left((h(x, y) \rho(x, y))^{\beta}\right)=$ $\varphi(h(x, y) \rho(x, y))$ with $\varphi(r)=\psi^{-1}\left(r^{\beta}\right)=\eta\left(r^{\beta}\right)$.

Notice that since $h$ is symmetric and bounded above and below by positive constants the function $d(x, y)=h(x, y) \rho(x, y)$ is a quasi-metric. But actually $d$ is better than a general quasi-metric since its triangular inequality constant can be taken to be independent of the length of chains. Precisely, $d\left(x_{1}, x_{m}\right) \leq$ $2^{2+1 / \beta} \sum_{j=1}^{m-1} d\left(x_{j}, x_{j+1}\right)$.

Let us observe also that Newtonian type power laws are obtained when $v(\lambda)=a \lambda$ for $a<1$. In fact, with $m=\frac{1}{\log _{2} a}<0, \psi(r)=r^{m}$ solves the equation $(a \lambda)^{m}=2 \lambda^{m}$. Hence $\varphi$ becomes also a power law.

Let us finally state and prove that the conditions on $K$ are necessary to have a Newtonian structure for $K$.

Proposition 4.2. Let $(X, d)$ be a quasi-metric space with triangular constant $\tau$. Let $\varphi$ be a positive function defined on $\mathbb{R}^{+}$such that, $\varphi$ is $\mathcal{C}^{2}\left(\mathbb{R}^{+}\right)$, convex, decreasing, $\varphi\left(0^{+}\right)=+\infty$ and $\varphi(+\infty)=0$. Let $K: X \times X \rightarrow \mathbb{R}^{+}$be given by $K(x, y)=\varphi(d(x, y))$. Then there exists a positive concave function $v$ defined on $\mathbb{R}^{+}$increasing, $v(0)=0$, $v(+\infty)=+\infty$ such that if $K(x, z)>\lambda>0$ and $K(z, y)>\lambda$ for some $z \in X$, we have that $K(x, y)>v(\lambda)$ with $v(\lambda)=\varphi\left(2 \tau \varphi^{-1}(\lambda)\right)$. Moreover, $v(\lambda)<\lambda$ for every $\lambda>0$.

Proof. Let $x, y$, and $z$ be as in the statement. In particular, $d(x, z)<\varphi^{-1}(\lambda)$ and $d(z, y)<\varphi^{-1}(\lambda)$. Hence

$$
d(x, y) \leq \tau(d(x, z)+d(z, y))<2 \tau \varphi^{-1}(\lambda) .
$$

So that $K(x, y)>\varphi\left(2 \tau \varphi^{-1}(\lambda)\right)=v(\lambda)$. Let us check that the function $v$ satisfies the required properties. Notice first that $v(\lambda)<\lambda$ follows directly from the decreasing of $\varphi$. The derivative of $v$ is given by

$$
\nu^{\prime}(\lambda)=\varphi^{\prime}\left(2 \tau \varphi^{-1}(\lambda)\right) \frac{2 \tau}{\varphi^{\prime}\left(\varphi^{-1}(\lambda)\right)} .
$$

Hence $v^{\prime}>0$ for every $\lambda>0$ and $v$ is increasing. The facts that $v\left(0^{+}\right)=0$ and $v(+\infty)=+\infty$ follows from $\varphi\left(0^{+}\right)=+\infty$ and $\varphi(+\infty)=0$. Let us finally check the concavity of $v$. Taking derivative with respect to $\lambda$ in the above formula for $v^{\prime}(\lambda)$ we see that

$$
v^{\prime \prime}(\lambda)=2 \tau \frac{\varphi^{\prime \prime}\left(2 \tau \varphi^{-1}(\lambda)\right) 2 \tau-\varphi^{\prime}\left(2 \tau \varphi^{-1}(\lambda)\right) \frac{\varphi^{\prime \prime}\left(\varphi^{-1}(\lambda)\right)}{\varphi^{\prime}\left(\varphi^{-1}(\lambda)\right)}}{\left[\varphi^{\prime}\left(\varphi^{-1}(\lambda)\right)\right]^{2}} .
$$

Set $s=\varphi^{-1}(\lambda)$, hence the sign of $v^{\prime \prime}$ is defined by the sign of $2 \tau \varphi^{\prime \prime}(2 \tau s)-\varphi^{\prime}(2 \tau s) \frac{\varphi^{\prime \prime}(s)}{\varphi^{\prime}(s)}$. Since $\left[\varphi^{\prime}(2 \tau s) \varphi^{\prime}(s)\right]^{\prime} \geq 0$ we see that

$$
\begin{aligned}
2 \tau \varphi^{\prime \prime}(2 \tau s) \varphi^{\prime}(s)-\varphi^{\prime}(2 \tau s) \varphi^{\prime \prime}(s) & =\left[\varphi^{\prime}(2 \tau s) \varphi^{\prime}(s)\right]^{\prime}-2 \varphi^{\prime \prime}(s) \varphi^{\prime}(2 \tau s) \\
& \geq-2 \varphi^{\prime \prime}(s) \varphi^{\prime}(2 \tau s)>0 .
\end{aligned}
$$

Acknowledgements: The authors gratefully acknowledge the comments of one of the referees who also pointed out the related results in the early paper [3] on metrizability of topological spaces.

The authors acknowledge the support of the CONICET, ANPCyT-MINCYT and UNL. Research partially supported by ANPCyT-MINCyT grant PICT-2015-3631.

\section{References}

[1] H. Aimar, L. Forzani, and R. Toledano Balls and quasi-metrics: a space of homogeneous type modeling the real analysis related to the Monge-Ampère equation. J. Fourier Anal. Appl., 4(4/5):377-381, 1998. 
[2] H. Aimar, B. laffei, and L. Nitti. On the Macías-Segovia metrization of quasi-metric spaces. Rev. Un. Mat. Argentina, 41(2):6775, 1998.

[3] E. W. Chittenden. On the metrization problem and related problems in the theory of abstract sets. Bull. Amer. Math. Soc., 33:13-34, 1927.

[4] R. R. Coifman, and S. Lafon. Diffusion maps Appl. Comput. Harmon. Anal., 21(1):5-30, 2006.

[5] R. R. Coifman, S. Lafon, A. B. Lee, M. Maggioni, B. Nadler, F. Warner, and S. W. Zucker. Geometric diffusions as a tool for harmonic analysis and structure definition of data: Diffusion maps. Proceedings of the National Academy of Sciences of the United States of America, 102(21):7426-7431, 2005.

[6] A. H. Frink. Distance functions and the metrization problem. Bull. Amer. Math. Soc., 43(2):133-142, 1937.

[7] J. L. Kelley. General topology. Springer-Verlag, New York-Berlin, 1975. Reprint of the 1955 edition [Van Nostrand, Toronto, Ont.], Graduate Texts in Mathematics, No. 27.

[8] R. A. Macías and C. Segovia. Lipschitz functions on spaces of homogeneous type. Adv. in Math., 33(3):257-270, 1979. 